\title{
Non-invasive Estimation of Pulsatile and Static Intracranial Pressure by Optical Coherence Tomography
}

Henrik Holvin Jacobsen

Oslo University Hospital-Ullevål

Øystein Kalsnes Jørstad

Oslo University Hospital-Ullevål

Morten C. Moe

Oslo University Hospital-Ullevål

Goran Petrovski

Oslo University Hospital-Ullevål

Are Hugo Pripp

Oslo University Hospital-Ullevål

Tiril Sandell

Vestre Viken Hospital

Per Kristian Eide ( $\nabla$ p.k.eide@medisin.uio.no)

University of Oslo

\section{Research Article}

Keywords: Intracranial pressure, Non-invasive, optical coherence tomography

Posted Date: May 6th, 2021

DOI: https://doi.org/10.21203/rs.3.rs-480386/v1

License: (c) (i) This work is licensed under a Creative Commons Attribution 4.0 International License. Read Full License 


\section{Abstract}

This study examined whether optical coherence tomography (OCT) can be used to estimate the pulsatile and static intracranial pressure (ICP) in patients with idiopathic intracranial hypertension (IIH). Continuous overnight monitoring of the pulsatile and static ICP and a single OCT examination were performed in 20 patients with $\mathrm{IH}$ and in 12 reference subjects without cerebrospinal fluid disturbances. The ICP measurements were obtained using a parenchymal sensor. The pulsatile ICP was determined as the mean ICP wave amplitude (MWA) and the static ICP was determined as the mean ICP. The IIH group had increased overnight MWA and mean ICP scores, and OCT demonstrated anterior deflection of the peripapillary Bruch's membrane angle (pBA) and increased optic nerve head height (ONHH). There was a significant positive correlation between the $\mathrm{pBA}$ and $\mathrm{ONHH}$ and the overnight MWA scores, and between mean ICP and $\mathrm{ONHH}$. The pBA and the $\mathrm{ONHH}$ also predicted abnormal overnight MWA, but the predictive power of mean ICP was non-significant after adjusting for age and body mass index. This study suggest that the OCT parameters pBA and ONHH can predict abnormal MWA scores.

\section{Introduction}

Continuous monitoring of intracranial pressure (ICP) is a cornerstone in the diagnosis and management of a wide range of intracranial disorders, such as traumatic brain injury, intracranial hemorrhage and cerebrospinal fluid (CSF) disturbances. ${ }^{1}$ Still, ICP monitoring is invasive and requires insertion of a probe or catheter into the intracranial compartment, thereby carrying a risk of complications. ${ }^{2,3} \mathrm{~A}$ wide range of non-invasive ways of measuring the static and pulsatile components of ICP have been explored, ${ }^{1,4-11}$ but so far no methods have been found clinically suitable.

The eye is a potential window for indirect assessment of the ICP due to the direct communication between the intracranial and intraorbital subarachnoid spaces, by which the ICP directly affects the optic nerve and eye globe. ${ }^{12}$ Papilledema is a widely recognized indirect sign of intracranial hypertension. Recently, optical coherence tomography (OCT) has gained increasing interest as a potential tool for noninvasive ICP (nICP) assessment. ${ }^{13-15}$ The OCT methodology measures the reflection of back-scattered light and allows for detection and quantification of even subtle morphological changes in the retina and the optic nerve. ${ }^{16}$ In patients with idiopathic intracranial hypertension $(\mathrm{IIH}),{ }^{17}$ the mean ICP, measured using a telemetric parenchymal ICP probe, correlated with OCT measures of the optic nerve head. Moreover, in a pediatric study ${ }^{18}$ OCT measurements of the retina and the optic nerve head detected elevated mean ICP, measured with a subdural probe. However, with the exception of these studies, the published reports on estimating ICP from OCT have merely studied the relationship between OCT findings and the lumbar CSF opening pressure, ${ }^{13-15,19-22}$ which may differ from the ICP measured dynamically with an intracranial probe. ${ }^{1,23}$ In addition, the previous studies only examined the static ICP, even though ICP consists of both static and pulsatile ICP. The latter results from the pressure fluctuations created by the cardiac contractions. ${ }^{1}$ The pulsatile ICP may be expressed as the mean wave amplitude (MWA ${ }^{24}$ and 
has been reported to better reflect the pressure-volume reserve capacity (or intracranial compliance) than the static ICP. ${ }^{1,25}$

This study explored the value of OCT as a potential non-invasive tool for estimating the pulsatile ICP, determined as the MWA, and the mean ICP measured overnight in patients with IIH.

\section{Results}

Patients. The study cohort consisted of 20 patients fulfilling the diagnostic criteria of $\mathrm{IH}^{26}$ and 12 reference (REF) subjects who had undergone diagnostic work-up for tentative (CSF) circulation failure without evidence of such (Table 1). Patients were included during the study period from September 2018 to November 2020. The patient cohorts were comparable with regard to age and body mass index (BMI). However, the IIH group had a higher proportion of females than the REF group. 
Table 1

Information about demographics and ICP and OCT scores

\begin{tabular}{|c|c|c|c|}
\hline & $\mathrm{IIH}$ & REF & P-value \\
\hline Number & 20 & 12 & \\
\hline Gender (Female/Male) & $18 / 2$ & $2 / 10$ & $<0.001$ \\
\hline Age (years) & $32.3 \pm 9.6$ & $33.9 \pm 8.9$ & ns \\
\hline $\mathrm{BMI}\left(\mathrm{kg} / \mathrm{m}^{2}\right)$ & $30.4 \pm 5.1$ & $29.1 \pm 4.6$ & ns \\
\hline Papilledema (present/ not present) & $7 / 13$ & $0 / 12$ & $<0.001$ \\
\hline IOP (mmHg) & $13.2 \pm 3.3$ & $13.8 \pm 3.3$ & ns \\
\hline \multicolumn{4}{|l|}{ Overnight MWA } \\
\hline Average $(\mathrm{mmHg})$ & $6.7 \pm 2.0$ & $3.5 \pm 0.5$ & $<0.001$ \\
\hline Percentage $\geq 5 \mathrm{mmHg}$ & $70.6 \pm 20.9$ & $7.3 \pm 6.7$ & $<0.001$ \\
\hline \multicolumn{4}{|l|}{ Overnight mean ICP } \\
\hline Average $(\mathrm{mmHg})$ & $13.4 \pm 5.6$ & $6.8 \pm 4.9$ & $=0.003$ \\
\hline Percentage $\geq 15 \mathrm{mmHg}$ & $26.9 \pm 28.7$ & $8.1 \pm 17.8$ & ns \\
\hline \multicolumn{4}{|l|}{ OCT } \\
\hline pBA (degrees) & $-0.24 \pm 3.58$ & $-3.03 \pm 2.76$ & $=0.038$ \\
\hline $\mathrm{ONHH}(\mu \mathrm{m})$ & $520.0 \pm 139.1$ & $385.2 \pm 116.5$ & $=0.013$ \\
\hline Mean mGCC $(\mu \mathrm{m})$ & $94.1 \pm 12.7$ & $98.9 \pm 6.2$ & ns \\
\hline Mean peripapillary RNFL $(\mu \mathrm{m})$ & $122.7 \pm 76.0$ & $101.0 \pm 12.9$ & ns \\
\hline Mean peripapillary TRT $(\mu \mathrm{m})$ & $335.7 \pm 67.8$ & $322.8 \pm 11.7$ & ns \\
\hline $\mathrm{RNFL/mGCC}$ & $1.2 \pm 0.6$ & $1.0 \pm 0.1$ & ns \\
\hline TRT/mGCC & $3.5 \pm 0.5$ & $3.3 \pm 0.2$ & ns \\
\hline \multicolumn{4}{|c|}{$\begin{array}{l}\text { BMI = body mass index; ICP = intracranial pressure; IIH = idiopathic intracranial hypertension; REF = } \\
\text { reference; IOP = intraocular pressure; MWA = mean ICP wave amplitude; mGCC = macular ganglion cell } \\
\text { complex; OCT = optical coherence tomography; ONHH = optic nerve head height; pBA = peripapillary } \\
\text { Bruch's angle; RNFL = peripapillary retinal nerve fiber layer; TRT = peripapillary total retinal thickness; } \\
\mu m=\text { micrometer. Numbers given as mean } \pm \text { standard deviation for continuous variables. Significant } \\
\text { differences determined by independent samples t-test for continuous data and by Pearson Chi-square } \\
\text { test for categorized data (ns = non-significant). }\end{array}$} \\
\hline
\end{tabular}

Comparison of ICP and OCT parameters between the groups. The IIH group showed both significantly higher overnight pulsatile ICP (MWA) and static ICP (mean ICP). The intraocular pressure did not differ between the groups (Table 1). According to our previously described upper normal threshold values for 
MWA and mean ICP, 27,28 the MWA was abnormal in 19 of 20 (95\%) patients in the IIH group but none of the REF subjects. The mean ICP was abnormal in 8 of 20 (40\%) IIH patients and 1 of 12 (8\%) REF subjects.

Regarding the OCT parameters, the peripapillary Bruch's angle (pBA) was significantly smaller in the IIH than the REF group, demonstrating a more anterior deflected peripapillary Bruch's membrane. The optic nerve head height $(\mathrm{ONHH})$ was significantly increased in the IIH group (Table 1). The other OCT parameters did not differ significantly between IIH and REF (Table 1).

Association between ICP and OCT parameters. There was a positive correlation between the OCT parameters pBA and $\mathrm{ONHH}$ and the ICP scores. In particular, the pBA became increasingly smaller (anteriorly deflected) with increasing pulsatile ICP, expressed as overnight average MWA (Fig. 1a) or percentage proportion of MWA $\geq 5 \mathrm{mmHg}$ (Fig. 1b). This was not significant for static ICP (mean ICP; Figs. 1c-d). Furthermore, there was a significant positive correlation between $\mathrm{ONHH}$ and overnight average MWA (Fig. 2a), proportion of MWA $\geq 5 \mathrm{mmHg}$ (Fig. 2b), and overnight mean ICP (Fig. 2c). The proportion of mean ICP $\geq 15 \mathrm{mmHg}$ did not correlate with $\mathrm{ONHH}$ (Fig. 2d).

Ability of OCT to predict abnormal pulsatile and static ICP. The ability of the OCT parameters PBA and $\mathrm{ONHH}$ to predict abnormal pulsatile and static ICP is presented in Table 2. Both pBA and ONHH predicted abnormal MWA, with optimal cut-points of $-0.65^{\circ}$ and $405 \mu \mathrm{m}$. Their predictive ability remained significant after adjusting for age and BMI (Table 3). The ONHH predicted mean ICP with optimal cut point of $517 \mu \mathrm{m}$ (Table 2), but this was non-significant after adjusting for age and BMI (Table 3). Accordingly, both the pBA and ONHH most accurately predicted the MWA scores. Figure 3 displays the receiver operating curves (ROCs). 
Table 2

The ability of OCT to predict elevated MWA and mean ICP

\begin{tabular}{|c|c|c|c|c|c|c|}
\hline OCT & ICP & $\begin{array}{l}\text { Crude estimate } \\
\text { OR }(95 \% \mathrm{Cl}), p \text { - } \\
\text { value }\end{array}$ & $\begin{array}{l}\text { AUC (95\% } \\
\mathrm{Cl})\end{array}$ & $\begin{array}{l}\text { Optimal } \\
\text { cut point }\end{array}$ & $\begin{array}{l}\text { Sensitivity at } \\
\text { cut point }\end{array}$ & $\begin{array}{l}\text { Specificity at } \\
\text { cut point }\end{array}$ \\
\hline $\mathrm{pBA}$ & $\begin{array}{l}\text { MWA } \\
\text { (elevated) }\end{array}$ & $\begin{array}{l}1.51(1.09- \\
2.08),=0.013\end{array}$ & $\begin{array}{l}0.82 \\
(0.66- \\
0.98)\end{array}$ & $-0.65^{\circ}$ & 0.75 & 0.92 \\
\hline $\mathrm{ONHH}$ & & $\begin{array}{l}1.01(1.00- \\
1.02),=0.007\end{array}$ & $\begin{array}{l}0.84 \\
(0.70- \\
0.99)\end{array}$ & $405 \mu \mathrm{m}$ & 0.88 & 0.69 \\
\hline $\mathrm{pBA}$ & $\begin{array}{l}\text { Mean ICP } \\
\text { (elevated) }\end{array}$ & $\begin{array}{l}1.35(0.96- \\
1.91), \mathrm{ns}\end{array}$ & $\begin{array}{l}0.72 \\
(0.53- \\
0.91)\end{array}$ & $-0.78^{\circ}$ & 0.86 & 0.64 \\
\hline $\mathrm{ONHH}$ & & $\begin{array}{l}1.01(1.00- \\
1.02),=0.007\end{array}$ & $\begin{array}{l}0.79 \\
(0.62- \\
0.96)\end{array}$ & $517 \mu \mathrm{m}$ & 0.86 & 0.73 \\
\hline $\begin{array}{l}\text { ICP }=\text { ir } \\
\text { optic n } \\
\text { ratio; C } \\
\text { determ } \\
\text { confid }\end{array}$ & $\begin{array}{l}\text { cranial pre } \\
\text { head heig } \\
\text { onfidence } \\
\text { by logisti } \\
\text { intervals. }\end{array}$ & $\begin{array}{l}\text { MWA = mean } \\
\text { CT = optical coh } \\
\text { al; ns = non-sig } \\
\text { ession analysis }\end{array}$ & $\begin{array}{l}\text { e amplitu } \\
\text { nce tome } \\
\text { cant. The } \\
\text { cluding d }\end{array}$ & $\begin{array}{l}\mathrm{pBA}=\mathrm{pe} \\
\text { phy; AUC } \\
\text { dictive a } \\
\text { minatior }\end{array}$ & $\begin{array}{l}\text { illary Bruch's } \\
\text { ea under the } \\
\text { of the OCT } p \\
\text { R and AUC n }\end{array}$ & $\begin{array}{l}\text { gle; } \mathrm{ONHH}= \\
\text { ve; OR = odds } \\
\text { ameters was } \\
95 \%\end{array}$ \\
\hline
\end{tabular}

Table 3

The age and BMI adjusted ability of OCT to predict elevated MWA and mean ICP

\begin{tabular}{|c|c|c|c|}
\hline \multirow[t]{2}{*}{ ОСТ } & \multirow[t]{2}{*}{ ICP } & Crude estimate & \multirow[t]{2}{*}{ AUC } \\
\hline & & OR (95\% Cl), p-value & \\
\hline pBA & \multirow{2}{*}{$\begin{array}{l}\text { MWA } \\
\text { (elevated) }\end{array}$} & $1.98(1.17-3.36),=0.011$ & $0.85(0.68-1.00)$ \\
\hline $\mathrm{ONHH}$ & & $1.01(1.00-1.02),=0.010$ & $0.86(0.72-0.99)$ \\
\hline pBA & \multirow[t]{2}{*}{ Mean ICP (elevated) } & $1.19(0.79-1.81), \mathrm{ns}$ & $0.79(0.58-0.99)$ \\
\hline $\mathrm{ONHH}$ & & $1.01(1.00-1.02), \mathrm{ns}$ & $0.84(0.68-1.00)$ \\
\hline \multicolumn{4}{|c|}{$\begin{array}{l}\text { ICP = intracranial pressure; } \mathrm{MWA}=\text { mean wave amplitude; } \mathrm{pBA}=\text { peripapillary Bruch's angle; } \mathrm{ONHH}= \\
\text { optic nerve head height; } \mathrm{OCT}=\text { optical coherence tomography; } \mathrm{AUC}=\text { area under the curve; } \mathrm{OR}=\text { odds } \\
\text { ratio; } \mathrm{Cl}=\text { confidence interval; } \mathrm{ns}=\text { non-significant; } \mathrm{BMI}=\text { body mass index. The age and } \mathrm{BMI} \text { adjustec } \\
\text { predictive ability of the OCT parameters was determined by logistic regression analysis, including } \\
\text { determination of OR and the AUC with } 95 \% \text { confidence intervals. }\end{array}$} \\
\hline
\end{tabular}

\section{Discussion}

This study investigated whether OCT may be used to non-invasively estimate the pulsatile and static ICP. The main observations were that the OCT parameters pBA and the ONHH correlated with the overnight 
pulsatile ICP (MWA) scores, while the other OCT parameters investigated did not differ between IIH and REF. The optimal cut-off values for the pBA could predict elevated MWA with a sensitivity and a specificity of $75 \%$ and $92 \%$. Similarly, the ONHH could predict elevated MWA with a sensitivity of $88 \%$ and specificity of $69 \%$. The OCT parameters did, however, not reliably predict the static ICP (mean ICP).

This study included both a patient group with $\mathrm{IIH}$ diagnosed according to current criteria ${ }^{26}$ and a reference group without CSF disturbances. The groups were comparable with respect to age and BMI. Both the pulsatile and static ICP scores were increased in the IIH group. An abnormal MWA in the majority of the IIH patients (95\%) and mean ICP being elevated in less than half of the subjects (40\%) compares to a previous study of $\mathrm{IH}$ patients with failed conservative and medical treatment. ${ }^{28}$ The MWA shows a better correlation with the intracranial compliance (i.e., intracranial pressure volume reserve capacity) than mean ICP, ${ }^{25}$ and intracranial compliance can be impaired despite normal mean ICP. ${ }^{29}$ Accordingly, $\mathrm{IIH}$ patients can have impaired intracranial compliance even though mean ICP is within normal thresholds. Another possible reason for the abnormal pulsatile ICP may be alterations at the capillary level, which may underlie the impaired glymphatic function (i.e. impaired paravascular molecular transport) that was recently demonstrated in IIH patients. ${ }^{30}$

The ICP scores were derived from continuous overnight measurements. Previous studies have explored various types of continuous non-invasive source signals to estimate ICP. ${ }^{4-7}$ Continuous monitoring of the optic nerve with OCT is, to our knowledge, not available. Yet, a single time point examination may be used to estimate the presence of normal or abnormal ICP. Thus, OCT could serve as a method to differentiate elevated from non-elevated pulsatile ICP. A definite diagnosis of abnormal pulsatile and static ICP still requires an invasive procedure.

The pBA was significantly smaller in the IIH group than the REF group. A smaller angle implies a more anterior position of the peripapillary Bruch's membrane. The position of this anatomical area reflects the balance between the pressure in the perioptic subarachnoid space and the intraocular pressure. ${ }^{31}$ Elevated ICP relative to the intraocular pressure causes an anterior deflection. Lowering ICP has been shown to reverse the anterior deflection. ${ }^{19,31,32}$ Illustrating the highly dynamic properties of this parameter, one study observed reversing of the position of the peripapillary Bruch's membrane within one hour after lowering the ICP. ${ }^{20}$ Previous studies have also shown anterior deflection of the Bruch's membrane in patients with elevated lumbar CSF opening pressure. ${ }^{19,21,31,33}$ However, contradictory to previous findings $20,22,34$, we did not find any correlation between the anterior deflection and the mean ICP. There are several potential reasons for this discrepancy. Both the lumbar CSF opening pressure and the mean ICP refer to the static ICP component; these pressures are not identical, as has been previously shown by simultaneous measurements. ${ }^{23}$ Moreover, a lumbar CSF pressure is measured over a very short period and may be extensively affected by stress or body position. The ICP scores presented in this study, however, were retrieved from multi-hour overnight measurements. The patients were resting in their beds and allowed to sleep. 
Our observation of a significant correlation between pBA and MWA indicates that the abnormal pulsatile pressure fluctuations during the cardiac beat are more susceptible to cause anterior deviation of the peripapillary Bruch's membrane than a static pressure gradient. Accordingly, repetitive small cardiacrelated pressure fluctuations (in the IIH group the average overnight MWA was $6.7 \pm 2.0 \mathrm{mmHg}$ ) seem to exert a different effect on the soft tissue than a static difference in pressure (the overnight mean ICP in IIH subjects was $13.4 \pm 5.6 \mathrm{mmHg}$ ).

We did not find between-group differences in the RNFL. Automatic algorithm for measuring the RNFL thickness is available in all OCT devices and widely used in the diagnosis and management of papilledema. ${ }^{13,14,35}$ Elevated ICP compresses the optic nerve and leads to axoplasmatic stasis and swelling of the retinal nerve fibers. ${ }^{12}$ However, since dead axons don't swell, a major drawback of measuring the RNFL is the potentially false impression of a normal or even thin RNFL in patients with increased ICP, if optic nerve atrophy has developed. Notably, the pBA is not affected by optic atrophy. Accordingly, it may be a more reliable biomarker for nICP estimation if atrophy has developed. Intriguing in this regard is a previous case report. ${ }^{19}$ This suggested the anterior deflection of the peripapillary Bruch's membrane to better indicate elevated ICP than the RNFL per se in a patient with optic nerve atrophy treated with CSF diversion (shunt) surgery.

The $\mathrm{ONHH}$ was higher in the IIH than the REF group. Elevated $\mathrm{ONHH}$ has been shown to correlate with the optic nerve head volume and likely reflects the swelling and protrusion of the optic nerve head caused by the increased ICP. ${ }^{14}$ The increased $\mathrm{ONHH}$ in the IIH group may reflect papilledema, though papilledema would expectedly be accompanied by increased thickness of the RNFL. ${ }^{14,36}$ Thus, the elevated ONHH in the absence of significantly elevated RNFL thickness observed in the IIH group may also be due to chronic changes like overlying gliosis. ${ }^{37}$ Previous studies of $\mathrm{ONHH}$ in relationship to ICP vary. Two studies ${ }^{17,18}$ demonstrated a correlation between the height of the optic nerve head and invasively measured mean ICP. Similarly, a second study found a trend towards a decrease in $\mathrm{ONHH}$ after lowering the CSF opening pressure. ${ }^{38}$ Other studies, however, found no ${ }^{14}$ or even a negative ${ }^{39}$ association between the $\mathrm{ONHH}$ and the lumbar CSF opening pressure.

Some limitations of this study should be noted. First, due to the lack of an automatic algorithm in the NIDEK software for pBA and ONHH determination, these may be examiner-dependent. To mitigate this potential bias, a consensus between two examiners was made. Second, for logistic reasons, we were not able to perform ICP and OCT measurements simultaneously. There was a time lag of weeks to months between the two examinations. However, we consider it less likely that the ICP or OCT scores would change significantly during this period. At least, the difference in time cannot explain the positive correlations between the independent variables. Third, the low number of study subjects is a limitation. Of note in this regard is the observed difference in the mean RNFL, which may be due to lack of statistical power.

\section{Conclusions}


The peripapillary Bruch's membrane angle and optic nerve head height correlated with the pulsatile ICP and differentiated between elevated and non-elevated ICP levels. Accordingly, our study provides proof of concept for a non-invasive method of detecting abnormal pulsatile ICP.

\section{Methods}

Approvals. The ICP scores are stored as part of a Neurovascular-Hydrocephalus quality registry approved by the Institutional Review Board of Oslo University Hospital (2011/6692). The OCT assessment was done as part of a study approved by the The Regional Committee for Medical and Health Research Ethics of Health Region South-East, Norway (2018/1074). The study was conducted according to the ethical standards of the Helsinki Declaration from 1975 and as revised in 1983. Informed consent was given by the participants prior to inclusion.

Design. A prospective observational study design was used. In order to examine how OCT parameters may be used to estimate pulsatile and static ICP, we 1) compared OCT and ICP scores between two patient cohorts, and 2) correlated the OCT and ICP scores of individual patients, and 3) determined the predictive ability of OCT regarding ICP.

Patients. The first group included patients with a diagnosis of $\mathrm{IH}_{1}{ }^{26}$ who underwent ICP monitoring as work up for tentative shunt surgery due to failed conservative-medical treatment. The second group included an age-matched patient group that underwent ICP monitoring as pre-operative work-up for tentative CSF disturbance, but in whom no CSF disturbance was identified and no surgical intervention recommended. Both groups underwent overnight ICP monitoring at the Department of Neurosurgery, Oslo University Hospital - Rikshospitalet, Norway.

Overnight ICP monitoring. Monitoring of the ICP was performed overnight as part of routine clinical work up in order to diagnose and select patients for CSF diversion surgery. ${ }^{28}$ Continuous ICP waveforms were recorded at 100-200 Hz using an intracranial pressure sensor (Codman ICP MicroSensor, Codman, Johnson \& Johnson, Raynham, Massachusetts) placed 1-2 cm into the parenchyma through a burr hole in the frontal bone under local anesthetics. ${ }^{24}$ For the MWA, single heart beat induced pressure waves are identified by an automatic algorithm, and the pulse amplitude is calculated as the pressure difference between the diastolic and systolic pressures over 6 -second time windows. ${ }^{24}$ The mean ICP is the sum of all pressure measurements during the 6-second time windows divided by the number of measurements. With regard to our previously reported upper normal ICP scores, ${ }^{27,28}$ abnormal pulsatile ICP was defined by average overnight MWA scores $\geq 4 \mathrm{mmHg}$ and/or $\geq 5 \mathrm{mmHg}$ in $\geq 10 \%$ of the recording time, and abnormal static ICP was defined by overnight average mean ICP $\geq 15 \mathrm{mmHg}$ and/or $\geq 10 \%$ of measurements $\geq 15 \mathrm{mmHg}$.

\section{Ophthalmological examination and OCT imaging protocol}


All ophthalmic examinations were performed at the Department of Ophthalmology, Oslo University Hospital - Ullevål, Norway by an experienced ophthalmic nurse and HHJ. To avoid dependent data, only the "worst eye" defined by mean defect on threshold perimetry was included. Exclusion criteria were refraction error with spherical equivalent outside the range of -6.0 to +3.0 diopters, concurrent eye disorders affecting the retina or optic nerve, and OCT scans with artifacts or poor quality (signal strength index $<7$ of 10$)$.

Standard white-on-white threshold perimetry of the central $30^{\circ}$ was performed using an Octopus 900 perimeter (Haag-Streit AG, Koeniz, Switzerland) and the G-TOP program. The perimetry was performed by a trained ophthalmic nurse. Presence of fundoscopic papilledema was evaluated by HHJ and graded as present or not present.

Spectral-domain OCT scans were acquired in mydriasis using a RS-3000 OCT Advance (NIDEK Co. LTD, Singapore) and its Navis-EX software (NIDEK Co.).

The pBA (Fig. 4) and the maximum ONHH (Fig. 5) were measured manually on $6 \mathrm{~mm}$ horizontal B-scans traversing through the center of the optic disc. For both measurements, consensus was reached between two observers. Measurements of the pBA were performed in Adobe Photoshop (Adobe, San Jose, California). The peripheral sections of the nasal and temporal Bruch's membrane were used as reference. Reference lines were obtained by drawing lines parallel to the Bruch's membrane in the nasal and temporal periphery of the scan. The angle for which the central section of Bruch's membrane deviated from the reference lines was determined nasally and temporally; the mean of the two angles constitutes the pBA. The angle was denoted negative if deviating posteriorly (i.e. away from the vitreous) and positive if deviating anteriorly. For the $\mathrm{ONHH}$ measurement we used the tools in the Navis-EX software. A reference line was drawn across the scleral opening, connecting the nasal and temporal ends of the retinal pigment epithelium/Bruch's membrane layers. The $\mathrm{ONHH}$ was measured by a straight line to the highest point of the optic nerve head (Fig. 2). The OCT parameters mean RNFL, the mean peripapillary total retinal thickness (TRT), and the macular ganglion cell complex (mGCC) were also measured and compared between the groups. The mean RNFL thickness and the mean TRT were automatically measured by the Navis-EX software using a ring scan centered at the optic disc with a diameter of 3.45 $\mathrm{mm}$. The mean TRT was measured from the inner limiting membrane to the retinal pigment epithelium/ Bruch's membrane. The mean mGCC was measured automatically by the Navis-Ex software using the 9x9mm Macular Map program.

\section{Statistical analyses}

An independent t-test was used for the comparison of continuous data between the groups. Correlations between independent variables were determined by the Pearson correlation coefficient. Logistic regression models calculated the odds ratio for continuous exposure variables. The optimal cut-off point for a diagnostic test was calculated from the ROCs using the Liu method. SPSS version 27 (IBM Corporation, Armonk, NY) or Stata/SE version 15.0 (StataCrop LLX, College Station, TX) with the user- 
develop command cutpt ${ }^{40}$ was used for the statistical calculations. Accepted statistical significance was $\leq 0.05$.

\section{Data availability}

The data by which this paper is based is available upon reasonable request.

\section{Declarations}

\section{Acknowledgments}

We wish to thank the nurse staff at the Hydrocephalus outpatient clinic, Department of Neurosurgery, Oslo University Hospital - Rikshospitalet, and the clinical research-nurse at the Department of Ophthalmology, Oslo University Hospital-Ullevål.

\section{Author contributions}

Conception and design: PKE, MCM, ØKJ, HHJ, TS. Acquisition of data: PKE, MCM, ØKJ, HHJ, TS. Analysis and interpretation of data: AHP, PKE, MCM, ØKJ, HHJ, TS. Drafting the article: HHJ. Statistical analyses: AHP, PKE. Critical revision of the article: AHP, PKE, MCM, ØKJ, TS, GP. Approval of the final version of the article: All authors.

\section{Funding}

The study was funded by the South-Eastern Norway Regional Health Authority (reference 2018020), the Norwegian Association of the Blind and Partially Sighted, and the Norwegian Glaucoma Research Foundation. The sponsor or funding organizations had no role in the design or conduct of this research.

\section{Competing interests}

The authors declare no competing interests.

\section{References}

1 Evensen, K. B. \& Eide, P. K. Measuring intracranial pressure by invasive, less invasive or non-invasive means: limitations and avenues for improvement. Fluids Barriers CNS17, 34, doi:10.1186/s12987-02000195-3 (2020).

2 Koskinen, L. O., Grayson, D. \& Olivecrona, M. The complications and the position of the Codman MicroSensor $^{\text {TM }}$ ICP device: an analysis of 549 patients and 650 Sensors. Acta Neurochir (Wien)155, 21412148; discussion 2148, doi:10.1007/s00701-013-1856-0 (2013).

3 Binz, D. D., Toussaint, L. G. \& Friedman, J. A. Hemorrhagic Complications of Ventriculostomy Placement: A Meta-Analysis. Neurocritical Care10, 253, doi:10.1007/s12028-009-9193-0 (2009). 
4 Evensen, K. B., O’Rourke, M., Prieur, F., Holm, S. \& Eide, P. K. Non-invasive Estimation of the Intracranial Pressure Waveform from the Central Arterial Blood Pressure Waveform in Idiopathic Normal Pressure Hydrocephalus Patients. Scientific Reports8, 4714, doi:10.1038/s41598-018-23142-7 (2018).

5 Evensen, K. B., Paulat, K., Prieur, F., Holm, S. \& Eide, P. K. Utility of the Tympanic Membrane Pressure Waveform for Non-invasive Estimation of The Intracranial Pressure Waveform. Scientific Reports8, 15776, doi:10.1038/s41598-018-34083-6 (2018).

6 Ringstad, G. et al. Non-invasive assessment of pulsatile intracranial pressure with phase-contrast magnetic resonance imaging. PLoS One12, e0188896-e0188896, doi:10.1371/journal.pone.0188896 (2017).

7 Levinsky, A., Papyan, S., Weinberg, G., Stadheim, T. \& Eide, P. K. Non-invasive estimation of static and pulsatile intracranial pressure from transcranial acoustic signals. Medical Engineering \& Physics38, 477484, doi:https://doi.org/10.1016/j.medengphy.2016.02.009 (2016).

8 Koskinen, L.-O. D. et al. Can intracranial pressure be measured non-invasively bedside using a two-depth Doppler-technique? Journal of Clinical Monitoring and Computing31, 459-467, doi:10.1007/s10877-0169862-4 (2017).

9 Kimberly, H. H., Shah, S., Marill, K. \& Noble, V. Correlation of Optic Nerve Sheath Diameter with Direct Measurement of Intracranial Pressure. Academic Emergency Medicine15, 201-204, doi:https://doi.org/10.1111/j.1553-2712.2007.00031.x (2008).

10 Andersen, M. S., Pedersen, C. B. \& Poulsen, F. R. A new novel method for assessing intracranial pressure using non-invasive fundus images: a pilot study. Scientific Reports10, 13062, doi:10.1038/s41598-020-70084-0 (2020).

11 Koziarz, A. et al. Bedside Optic Nerve Ultrasonography for Diagnosing Increased Intracranial Pressure: A Systematic Review and Meta-analysis. Ann. Intern. Med., doi:10.7326/m19-0812 (2019).

12 Hayreh, S. S. Pathogenesis of optic disc edema in raised intracranial pressure. Prog Retin Eye Res50, 108-144, doi:10.1016/j.preteyeres.2015.10.001 (2016).

13 Malhotra, K., Padungkiatsagul, T. \& Moss, H. E. Optical coherence tomography use in idiopathic intracranial hypertension. Ann Eye Sci5, doi:10.21037/aes.2019.12.06 (2020).

14 Group, O. C. T. S.-S. C. f. N. I. I. H. S. et al. Baseline OCT measurements in the idiopathic intracranial hypertension treatment trial, part II: correlations and relationship to clinical features. Investigative ophthalmology \& visual science55, 8173-8179, doi:10.1167/iovs.14-14961 (2014).

15 Skau, M. et al. Diagnostic value of optical coherence tomography for intracranial pressure in idiopathic intracranial hypertension. Graefe's Archive for Clinical and Experimental Ophthalmology251, 567-574, doi:10.1007/s00417-012-2039-z (2013).

Page $12 / 18$ 
16 Huang, D. et al. Optical coherence tomography. Science254, 1178-1181, doi:10.1126/science.1957169 (1991).

17 Vijay, V. et al. Using Optical Coherence Tomography as a Surrogate of Measurements of Intracranial Pressure in Idiopathic Intracranial Hypertension. JAMA Ophthalmology, doi:10.1001/jamaophthalmol.2020.4242 \%J JAMA Ophthalmology (2020).

18 Swanson, J. W. et al. Evaluation of Optical Coherence Tomography to Detect Elevated Intracranial Pressure in Children. JAMA ophthalmology135, 320-328, doi:10.1001/jamaophthalmol.2017.0025 (2017).

19 Sibony, P., Kupersmith, M. J., Honkanen, R., Rohlf, F. J. \& Torab-Parhiz, A. Effects of lowering cerebrospinal fluid pressure on the shape of the peripapillary retina in intracranial hypertension. Invest Ophthalmol Vis Sci55, 8223-8231, doi:10.1167/iovs.14-15298 (2014).

20 Gampa, A., Vangipuram, G., Shirazi, Z. \& Moss, H. E. Quantitative Association Between Peripapillary Bruch's Membrane Shape and Intracranial Pressure. Invest Ophthalmol Vis Sci58, 2739-2745, doi:10.1167/iovs.17-21592 (2017).

21 Sibony, P., Kupersmith, M. J. \& Rohlf, F. J. Shape analysis of the peripapillary RPE layer in papilledema and ischemic optic neuropathy. Investigative ophthalmology \& visual science52, 7987-7995, doi:10.1167/iovs.11-7918 (2011).

22 Patel, M. D., Malhotra, K., Shirazi, Z. \& Moss, H. E. Methods for Quantifying Optic Disc Volume and Peripapillary Deflection Volume Using Radial Optical Coherence Tomography Scans and Association With Intracranial Pressure. Front Neuro/10, 798, doi:10.3389/fneur.2019.00798 (2019).

23 Eide, P. K. \& Brean, A. Lumbar cerebrospinal fluid pressure waves versus intracranial pressure waves in idiopathic normal pressure hydrocephalus. Br. J. Neurosurg.20, 407-414 (2006).

24 Eide, P. K. A new method for processing of continuous intracranial pressure signals. Medical Engineering \& Physics28, 579-587, doi:https://doi.org/10.1016/j.medengphy.2005.09.008 (2006).

25 Eide, P. K. The correlation between pulsatile intracranial pressure and indices of intracranial pressurevolume reserve capacity: results from ventricular infusion testing. J Neurosurg125, 1493-1503, doi:10.3171/2015.11.Jns151529 (2016).

26 Mollan, S. P. et al. Idiopathic intracranial hypertension: consensus guidelines on management. J. Neurol. Neurosurg. Psychiatry89, 1088-1100, doi:10.1136/jnnp-2017-317440 (2018).

27 Eide, P. K. \& Sorteberg, W. Diagnostic intracranial pressure monitoring and surgical management in idiopathic normal pressure hydrocephalus: a 6-year review of 214 patients. Neurosurgery66, 80-91, doi:10.1227/01.neu.0000363408.69856.b8 (2010). 
28 Eide, P. K. \& Kerty, E. Static and pulsatile intracranial pressure in idiopathic intracranial hypertension. Clin Neurol Neurosurg113, 123-128, doi:10.1016/j.clineuro.2010.10.008 (2011).

29 Eide, P. K. \& Sorteberg, W. Association among intracranial compliance, intracranial pulse pressure amplitude and intracranial pressure in patients with intracranial bleeds. Neurol. Res.29, 798-802, doi:10.1179/016164107x224132 (2007).

30 Eide, P. K., Pripp, A.H., Ringstad, G., Valnes, L.M. Impaired glymphatic function in idiopathic intracranial hypertension. Brain Commun (2021).

31 Kupersmith, M. J., Sibony, P., Mandel, G., Durbin, M. \& Kardon, R. H. Optical coherence tomography of the swollen optic nerve head: deformation of the peripapillary retinal pigment epithelium layer in papilledema. Invest Ophthalmol Vis Sci52, 6558-6564, doi:10.1167/iovs.10-6782 (2011).

32 Wang, J.-K. et al. Peripapillary Retinal Pigment Epithelium Layer Shape Changes From Acetazolamide Treatment in the Idiopathic Intracranial Hypertension Treatment Trial. Investigative Ophthalmology \& Visual Science58, 2554-2565, doi:10.1167/iovs.16-21089 \%J Investigative Ophthalmology \& Visual Science (2017).

33 Sibony, P. A. \& Kupersmith, M. J. Geometric Morphometrics Of The Peripapillary RPE Layer By OCT. Investigative Ophthalmology \& Visual Science52, 3004-3004 (2011).

34 Malhotra, K., Patel, M. D., Shirazi, Z. \& Moss, H. E. Association Between Peripapillary Bruch's Membrane Shape and Intracranial Pressure: Effect of Image Acquisition Pattern and Image Analysis Method, a Preliminary Study. Front Neuro/9, 1137, doi:10.3389/fneur.2018.01137 (2018).

35 Ophir, A., Karatas, M., Ramirez, J. A. \& Inzelberg, R. OCT and chronic papilledema. Ophthalmology112, 2238, doi:10.1016/j.ophtha.2005.09.004 (2005).

36 Karam, E. Z. \& Hedges, T. R. Optical coherence tomography of the retinal nerve fibre layer in mild papilloedema and pseudopapilloedema. The British journal of ophthalmology89, 294-298, doi:10.1136/bjo.2004.049486 (2005).

37 Ko, M. W. in Liu, Volpe, and Galetta's Neuro-Ophthalmology (Third Edition) (eds Grant T. Liu, Nicholas J. Volpe, \& Steven L. Galetta) 197-235 (Elsevier, 2019).

38 Anand, A. et al. Optical coherence tomography of the optic nerve head detects acute changes in intracranial pressure. Journal of Clinical Neuroscience29, 73-76, doi:10.1016/j.jocn.2015.12.016 (2016).

39 Kaufhold, F. et al. Optic nerve head quantification in idiopathic intracranial hypertension by spectral domain OCT. PLoS One7, e36965, doi:10.1371/journal.pone.0036965 (2012).

40 Clayton, P. CUTPT: Stata module for empirical estimation of cutpoint for a diagnostic test. Statistical Software Components (2013). 
a)

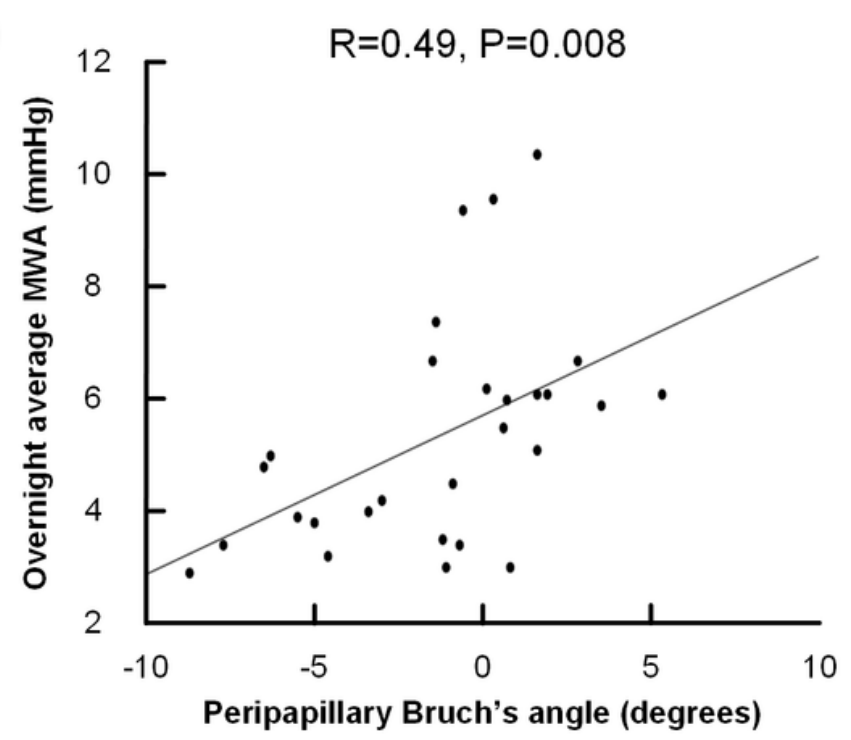

c)

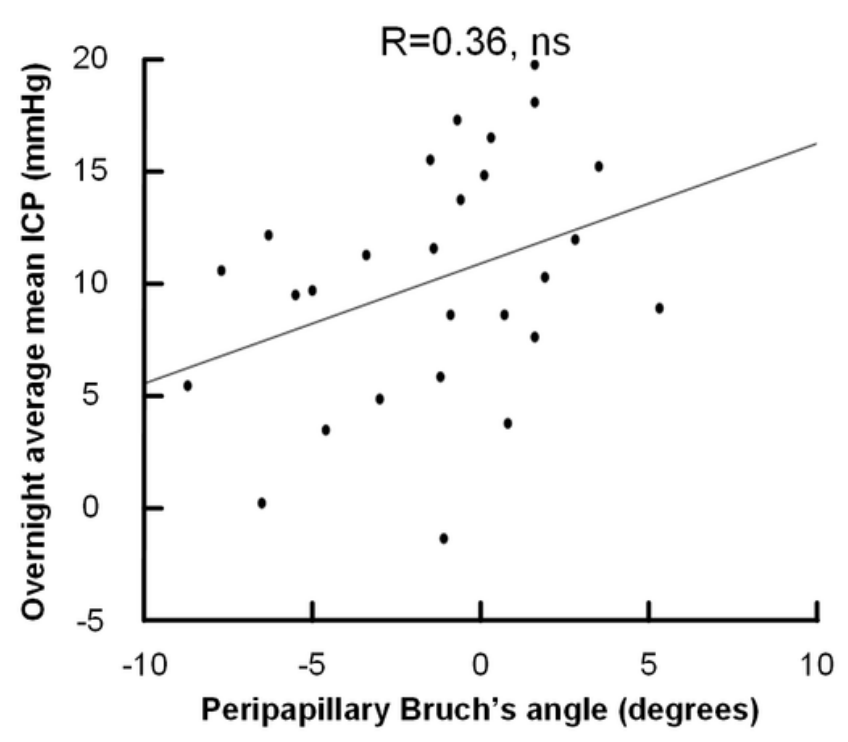

b)

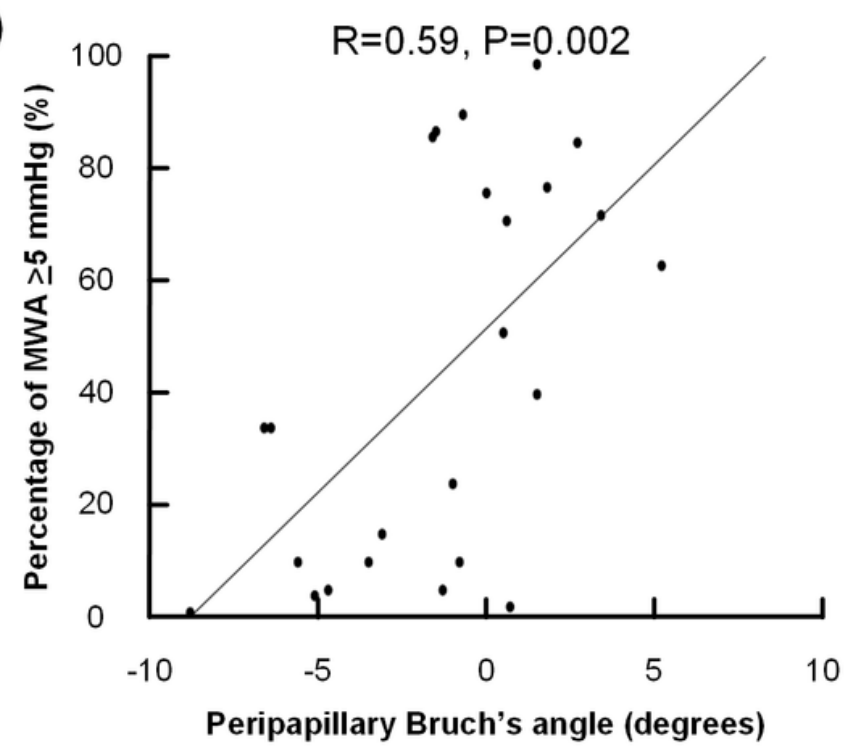

d)

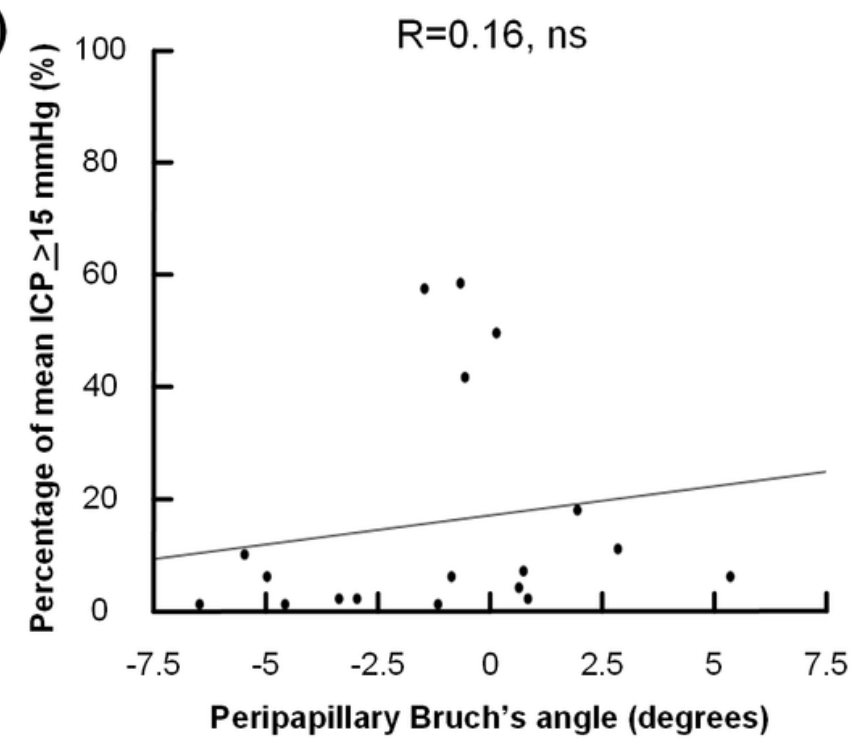

Figure 1

Association between the peripapillary Bruch's membrane angle (pBA) and pulsatile and static ICP scores. There was a significant positive correlation between (a) the peripapillary Bruch's membrane angle (pBA) and the average overnight mean ICP wave amplitude (MWA) and (b) between the pBA and the overnight percentage of MWA $\geq 5 \mathrm{mmHg}$. No significant correlation was found between $\mathrm{pBA}$ and (c) average of overnight mean intracranial pressure (ICP) or (d) overnight percentage mean ICP $\geq 15 \mathrm{mmHg}$. Each plot shows the fit line and Pearson correlation coefficient with significance levels (ns=non-significant). 
a)

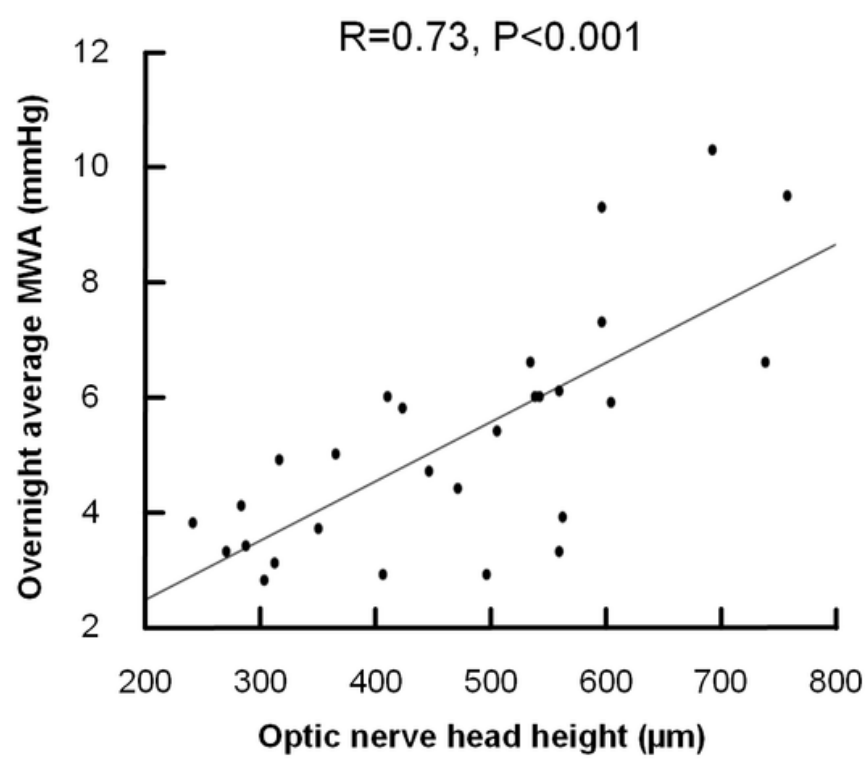

c)

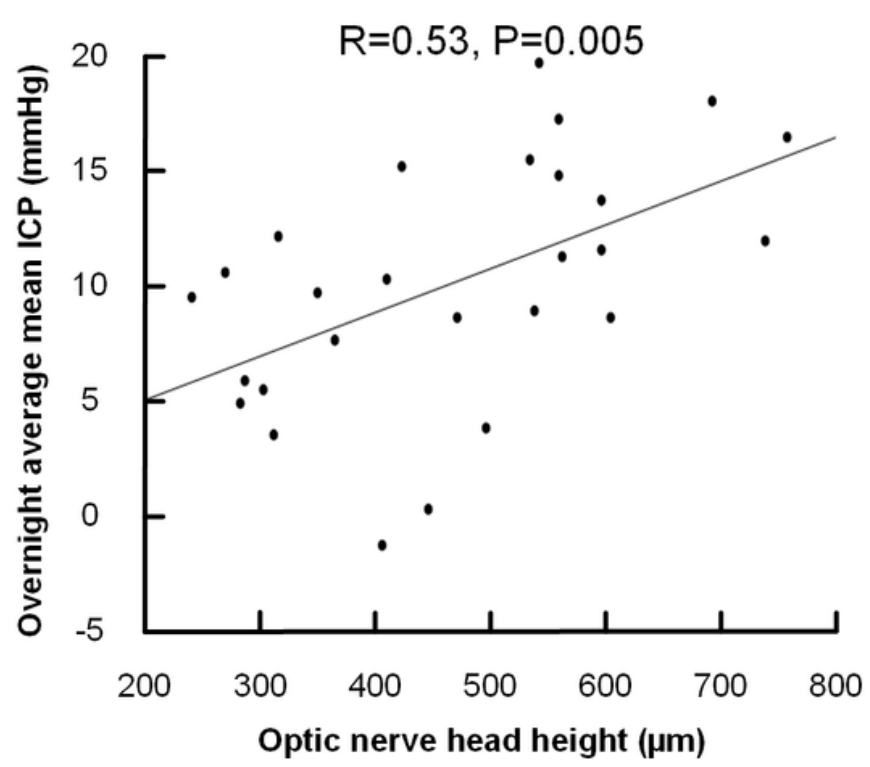

b)

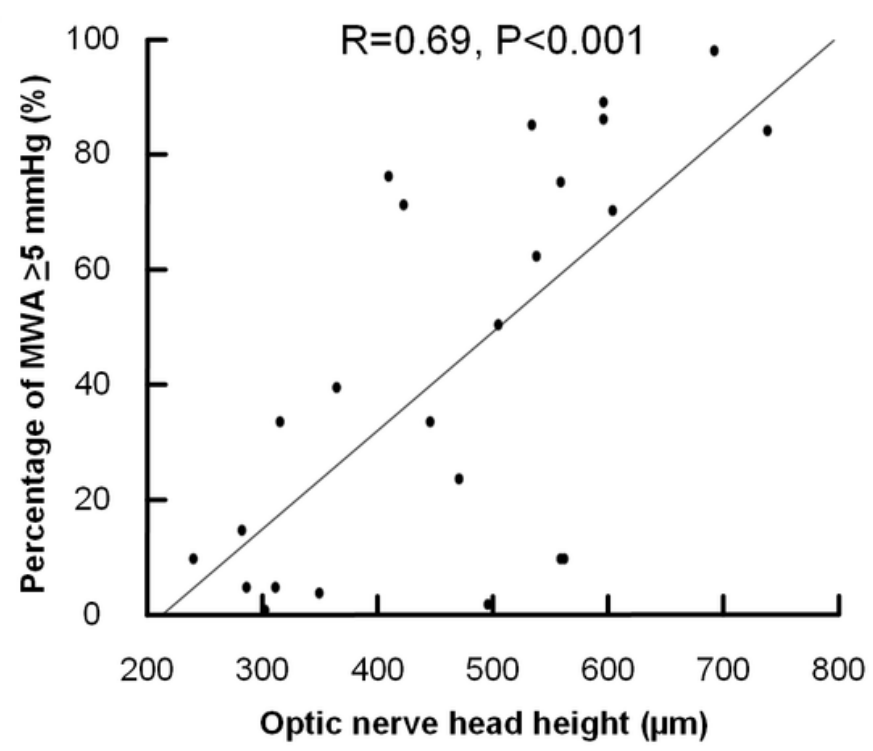

d)

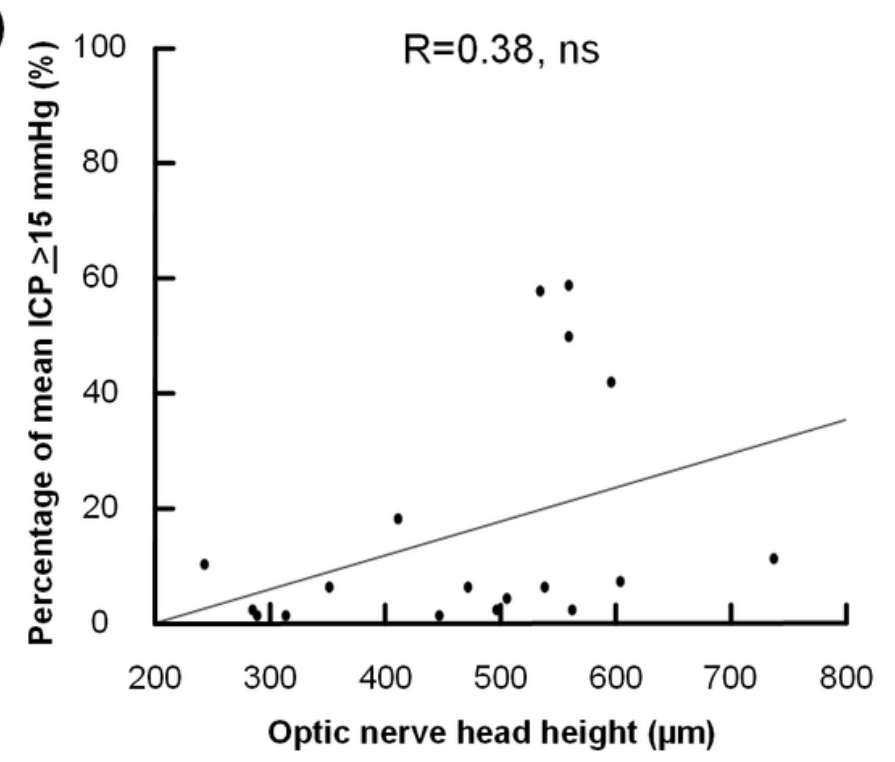

Figure 2

Association between the optic nerve head height $(\mathrm{ONHH})$ and pulsatile and static ICP scores. There was a significant positive correlation between (a) the optic nerve head height $(\mathrm{ONHH})$ and the average overnight mean ICP wave amplitude (MWA), and (b) between $\mathrm{ONHH}$ and the overnight percentage of MWA $\geq 5 \mathrm{~mm}$. There also was significant positive correlation between (c) $\mathrm{ONHH}$ and average of overnight mean intracranial pressure (ICP), but no correlation between (d) $\mathrm{ONHH}$ and overnight percentage mean ICP $\geq 15$ $\mathrm{mmHg}$. Each plot shows the fit line and Pearson correlation coefficient with significance levels (ns=nonsignificant). 
a)

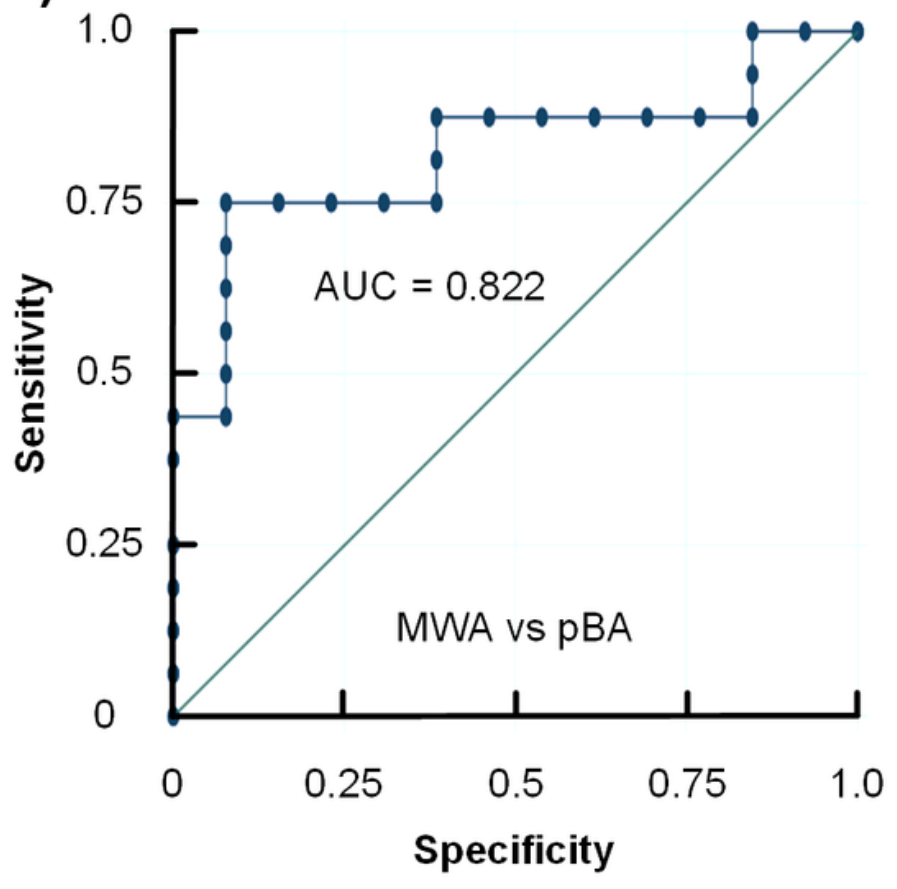

c)

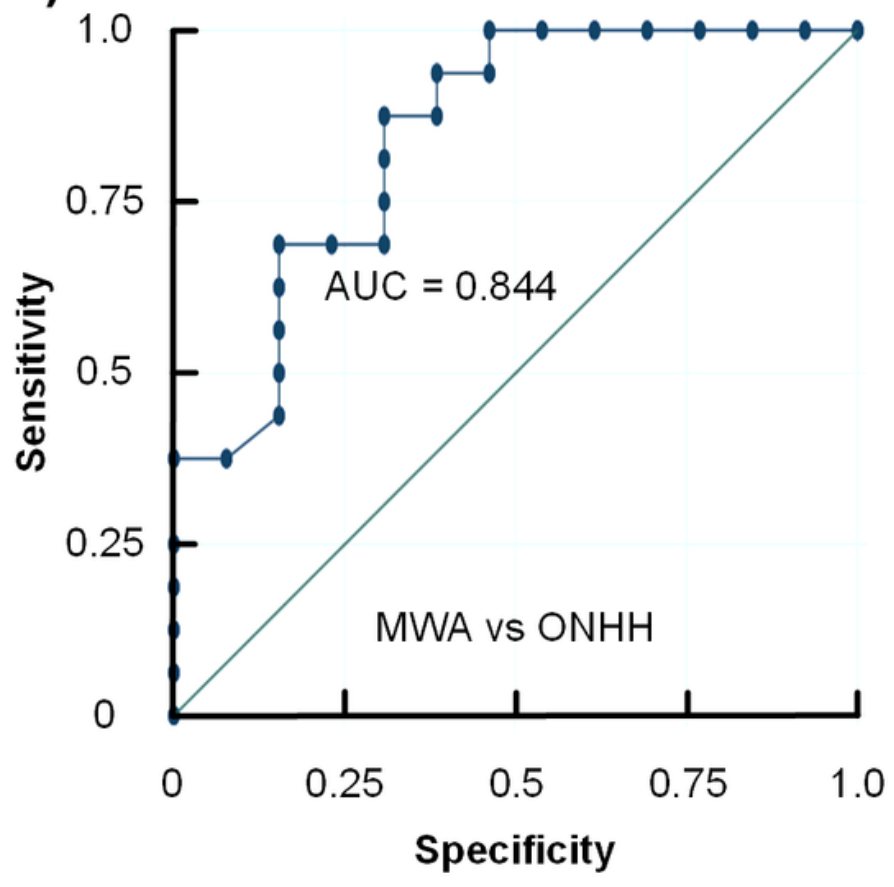

b)

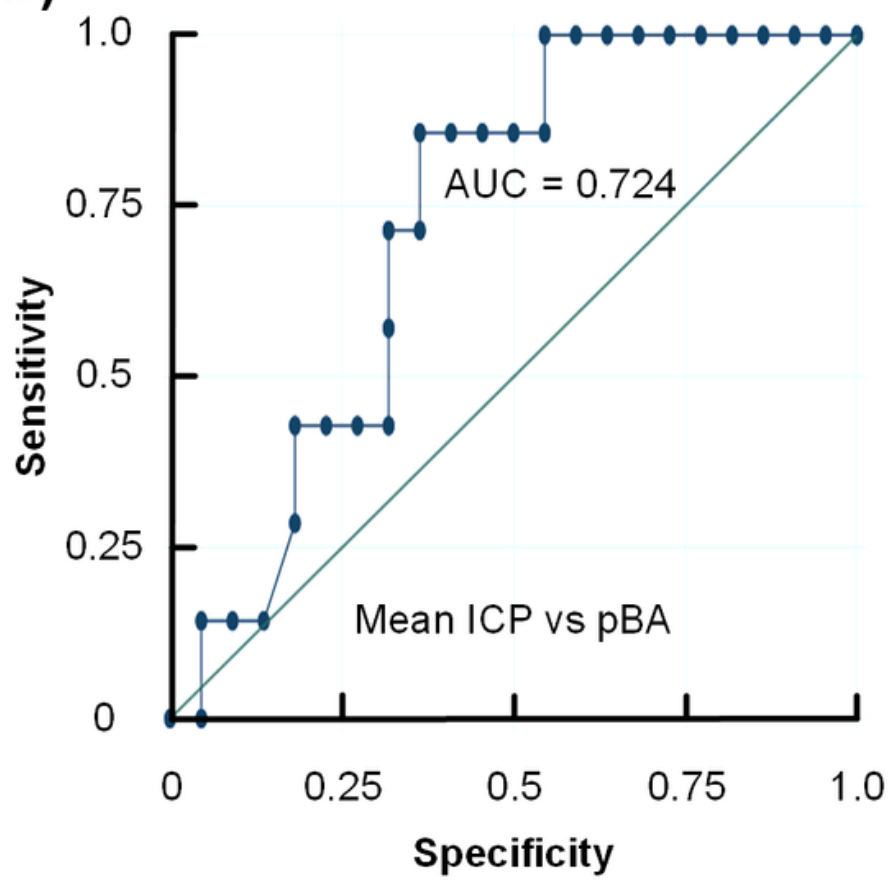

d)

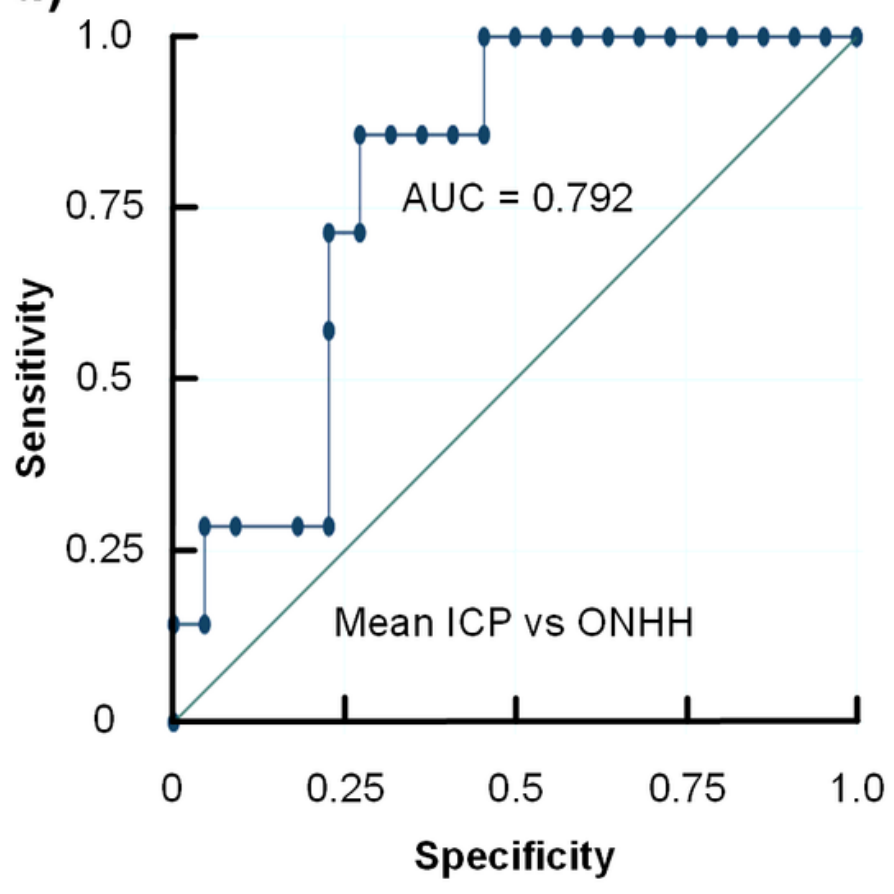

Figure 3

Receiver operating curves for ability of OCT parameters to predict ICP scores. The peripapillary Bruch's membrane angle (pBA) showed better ability to predict (a) the overnight mean wave amplitude (MWA) than (b) the overnight mean intracranial pressure (ICP). Likewise, the optic nerve head height (ONHH) showed better ability to predict (c) the overnight MWA than (d) the overnight mean ICP. For details about ROC results see Tables 2 and 3 . 


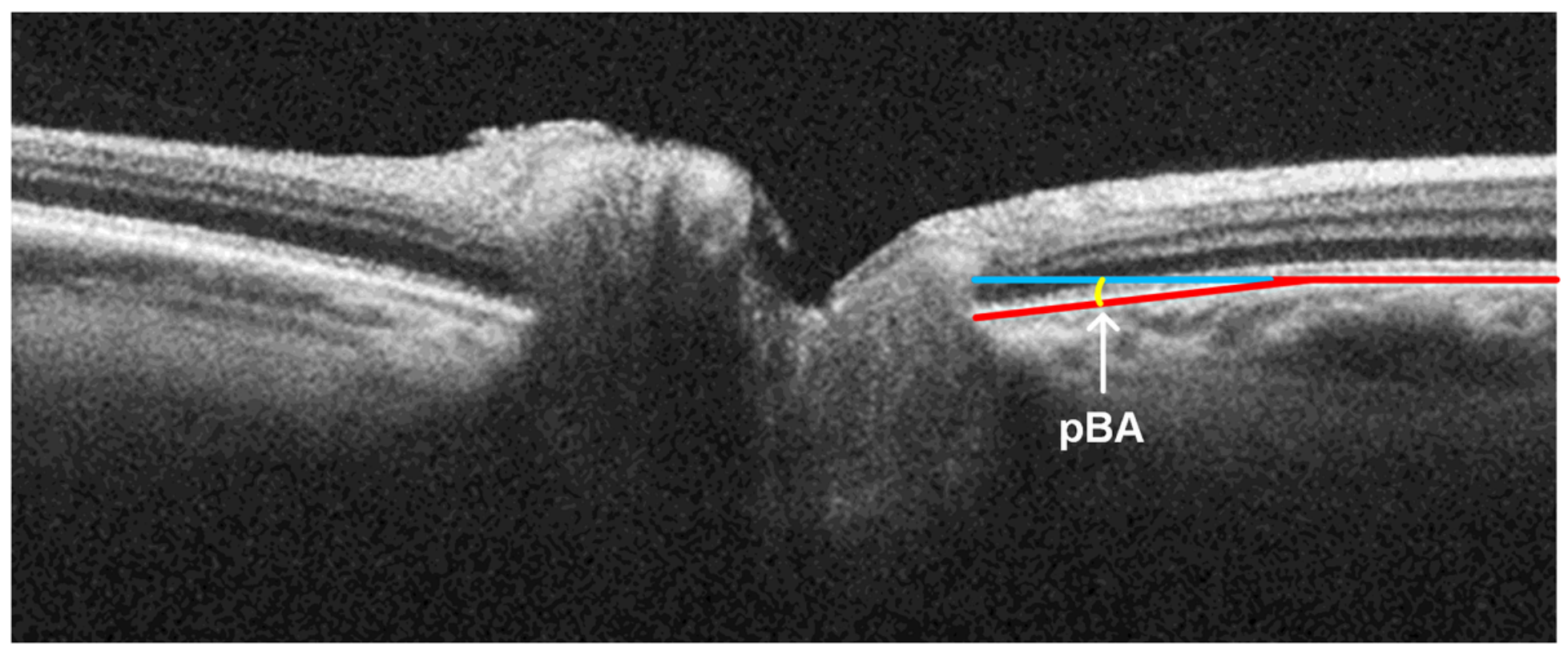

\section{Figure 4}

The peripapillary Bruch's membrane angle (pBA). The Bruch's membrane/ retinal pigment epithelium is marked by a red line. The peripapillary Bruch's membrane angle (pBA, yellow) is the angle which is formed between the deviated peripapillary Bruch's membrane (red line) and the unaltered part (blue line). A mean of nasal and temporal angles was calculated.

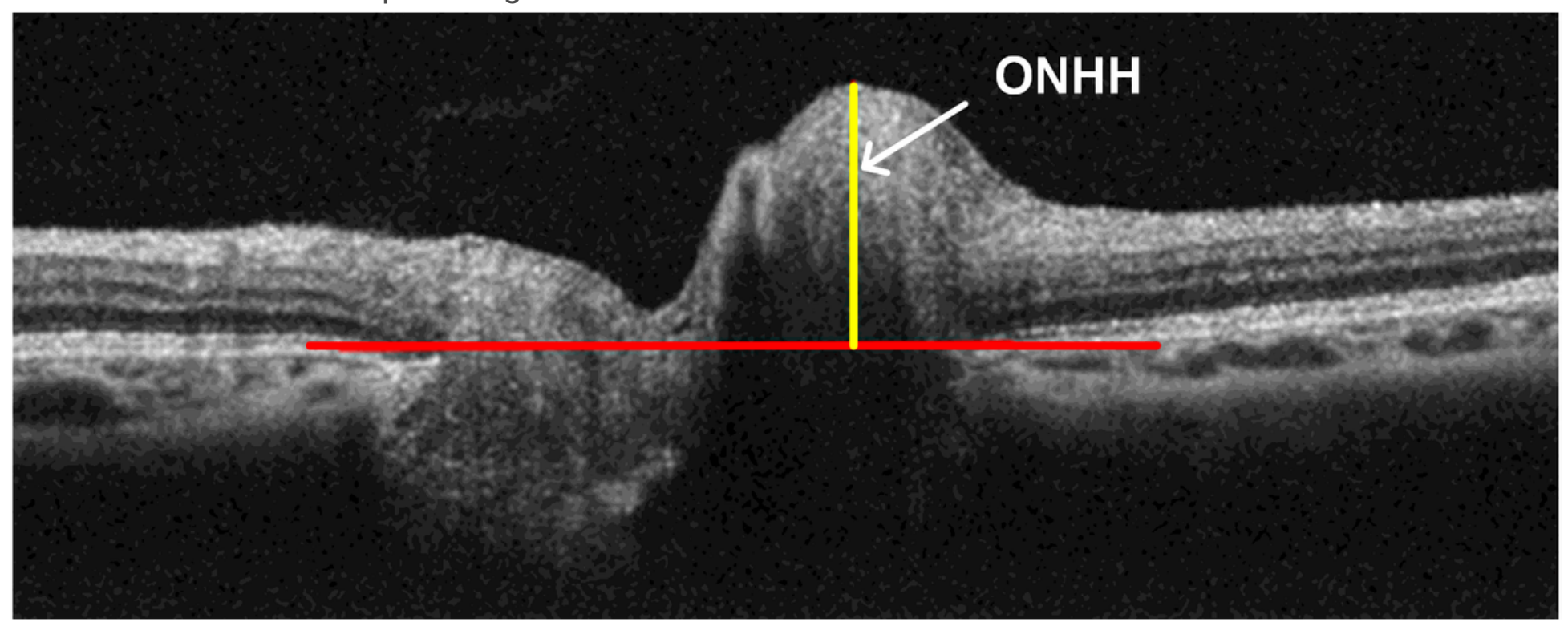

\section{Figure 5}

The optic nerve head height ( $\mathrm{ONHH}$ ). A line connecting both sides (nasal-temporal) of the scleral canal opening (red line) was used as a reference line. The height was measured from the highest point of the optic nerve head to the reference line (yellow line). 